\title{
Old ideas to innovate tuberculosis control: preventive treatment to achieve elimination
}

\author{
Roland Diel' ${ }^{1}$, Robert Loddenkemper², Jean-Pierre Zellweger ${ }^{3}$, Giovanni Sotgiu ${ }^{4}$, \\ Lia D’Ambrosio ${ }^{5}$, Rosella Centis ${ }^{5}$, Marieke J. van der Werf ${ }^{6}$, Masoud Dara7, \\ Anne Detjen ${ }^{8}$, Peter Gondrie $^{9}$, Lee Reichman ${ }^{10}$, Francesco Blasi $^{11}$ and \\ Giovanni Battista Migliori ${ }^{5}$ on behalf of the European Forum for TB Innovation
}

\begin{abstract}
Affiliations: 'Institute for Epidemiology, University Medical Hospital Schleswig-Holstein, Member of the German Center for Lung Research (DZL), Kiel, ${ }^{2}$ German Central Committee Against Tuberculosis, Berlin, Germany. ${ }^{3}$ Swiss Lung Association, Bern, Switzerland. ${ }^{4}$ Epidemiology and Medical Statistics Unit, Dept of Biomedical Sciences, University of Sassari, Sassari, ${ }^{5}$ World Health Organization Collaborating Centre for Tuberculosis and Lung Diseases, Fondazione S. Maugeri, Care and Research Institute, Tradate, "'Dipartimento Fisiopatologia Medico-Chirurgica e dei Trapianti, University of Milan, IRCCS Fondazione Cà Granda, Milan, Italy. ${ }^{6}$ European Centre for Disease Prevention and Control (ECDC), Stockholm, Sweden. ${ }^{7}$ World Health Organization, Regional Office for Europe, Copenhagen, Denmark. ${ }^{8}$ International Union Against Tuberculosis and Lung Disease, New York, NY, ${ }^{10}$ Global Tuberculosis Institute, New Jersey Medical School, Newark, NJ, USA. ${ }^{9}$ International Unit, KNCV Tuberculosis Foundation, The Hague, The Netherlands.
\end{abstract}

Correspondence: G.B. Migliori, World Health Organization Collaborating Centre for Tuberculosis and Lung Diseases, Fondazione S. Maugeri, Care and Research Institute, Via Roncaccio 16, 21049, Tradate, Italy. E-mail: giovannibattista.miglioridfsm.it

ABSTRACT The introduction of new rapid diagnostic tools for tuberculosis (TB) and the promising TB drugs pipeline together with the development of a new World Health Organization Strategy post 2015 allows new discussions on how to direct TB control. The European Respiratory Society's European Forum for TB Innovation was created to stimulate discussion on how to best take advantage of old and new opportunities, and advances, to improve TB control and eventually progress towards the elimination of TB.

While TB control is aimed at reducing the incidence of TB by early diagnosis and treatment of infectious cases of TB, TB elimination requires focus on sterilising the pool of latently infected individuals, from which future TB cases would be generated.

This manuscript describes the three core components that are necessary to implement the elimination strategy fully. 1) Improve diagnosis of latent TB infected individuals. 2) Improve regimens to treat latent TB infection. 3) ensure public health commitment to make both 1) and 2) possible. Old and new evidence is critically described, focusing on the European commitment to reach elimination and on the innovative experiences and best practices available.

@ERSpublications

Diagnosis and treatment of latent TB infection is the core intervention to reach elimination http://ow.ly/mjW0R 


\section{Introduction}

It is estimated that up to one-third of the world's population is latently infected with Mycobacterium tuberculosis and that the 10\% progression into active tuberculosis (TB) disease could be prevented by an effective preventive treatment [1] and represents a valid argument for complementing current TB control strategies [2-4] with innovative interventions focusing on the management of latent TB infection (LTBI).

On a TB-control level, the cure of infectious TB patients in the community has for decades represented the pillar of TB programmes, aiming at reducing transmission of M. tuberculosis in the community [5]. This approach, known as the directly observed treatment short course (DOTS), was incorporated into the World Health Organization's (WHO) "Stop TB Strategy" [6-8] and proved to be highly cost-effective [9-11]. Through proper diagnosis and rapid treatment of infectious cases the approach has saved millions of lives while breaking the chain of transmission, representing now the minimum package of interventions to tackle tuberculosis [11]. As existing strategies have not accelerated the progress towards elimination as much as previously expected $[12,13]$, WHO STAG-TB (Strategic and Technical Advisory Group for Tuberculosis) in June 2012 has initiated discussions on the future post-2015 strategy.

The term preventive treatment applies to the prevention and treatment of TB on an individual level [14, 15], rather than awaiting the symptomatic and active form of the disease to occur and implementing curative interventions. The public health impact of $\mathrm{TB}$ stimulates a shift from the individual implementation of this intervention to a programmatic and population-based approach.

The definitions of TB control and elimination are reported in table 1.

We are currently facing the stimulating introduction of new diagnostics [16], enabling a more rapid/ improved identification of drug-resistant tuberculosis (MDR-TB) cases and latently infected individuals, as well as new promising anti-TB drugs [17-20]. Complementing the high level policy work of WHO and international agencies, the European Respiratory Society (ERS) has launched the European Forum for TB Innovation in order to stimulate discussions on how to best take advantage of new opportunities and advances in improving TB control and, eventually, progress towards the elimination of [21].

In this perspective review, as part of the Forum activities, we summarise existing evidence on the programmatic feasibility and potential impact of the management of LTBI in the different high-risk groups for TB and the need to better assess its potential in accelerating the progression towards the elimination of TB on a global scale.

\section{From Zorini to Thibela trial and between: the TB preventive treatment approach}

Credit for the study and application of the concept of TB preventive treatment can be attributed to several investigators and physicians from both sides of the Atlantic. While the earliest reported and comprehensive theoretical discussion on the rational and use of preventive treatment was described, in 1957, in an article by ZORINI et al. [22], the first population-based implementation took shape under the research agenda of the US Public Health Service. In fact, the first efforts to sterilise LTBI on a large, population scale, were made using isoniazid (INH) preventive treatment (IPT) in a randomised controlled trial in household contacts in the Bethel district of Alaska (AK, USA), an area, which at that time, had one of the worlds highest TB incidences (578 per 100000 inhabitants) [23]. The study, which began in 1957, demonstrated a 69\% reduction in TB incidence comparing the treated group with the placebo group after 1 year and a community-wide IPT-related benefit with a sustained overall $30 \%$ reduction in TB incidence. This study was followed by several other studies in different risk groups, which all concluded that the prescription of preventative treatment, in subjects exposed to TB and latently infected, was beneficial and should be implemented on a larger scale [24]. The concept of a community-wide preventive-treatment intervention to sustain public-health goals faded progressively in its theoretical and practical application in the decades following the findings of the 1960s. A combination of discouraging requirements, from a logistical point of view, and the advent of a seemingly effective strategy based on finding infectious TB cases and curing them

\section{TABLE 1 Definitions of tuberculosis (TB) control and elimination}

TB control

Elimination
Strategy aimed at reducing the incidence of TB infection and, consequently, of TB disease, based on early diagnosis and treatment of infectious cases of TB. Fewer and fewer new people in the community will be exposed to a contact with the bacilli and will develop the disease

Point at which less than one infectious (sputum smear positive) case per 1000000 inhabitants emerges annually in the general population or when the prevalence of TB infection in general population is below $1 \%$ and continues to decrease

Table reproduced from [8]. 
slowly, turned TB preventive treatment into a secondary public-health intervention, mainly aimed at high risk groups.

However, the recent realisation that progress towards $\mathrm{TB}$ elimination requires the synergistic implementation of additional control measures has revived the idea of a programmatic role for TB preventive treatment.

Based on the experience of the early community-based trials two large innovative trials have been conducted. A randomised clinical trial in Brazil, Canada, Spain, and the USA, lasting up to 10 years, compared 12 doses of INH and rifapentine (RPT); given as weekly DOT with 9 months of self-administered INH given daily for 9 months [25]. Of the 7731 eligible subjects aged $\geqslant 2$ years a total of 3986 persons received the new combination, which proved to be both less toxic and more likely to be completed. As in the modified intention-to-treat analysis the cumulative proportion of subjects in which TB developed was $0.19 \%$ in the combination therapy group and $0.43 \%$ in the group given INH only, the new combination prevented TB in an at least equivalent percentage. Although the effect estimates of the study imply the potential for bias, due to the different strategy of drug administration in the study arms (self-administration versus DOT), the study resulted in a recent US Centers for Disease Control and Prevention (CDC) recommendation for use of an INH-RPT regimen in the USA [26].

The workplace-based Thibela TB trial among gold miners in South Africa, a group with a high prevalence of HIV infection and an estimated LTBI prevalence of $89 \%$ [27], was led by the Aurum Institute as part of the CREATE consortium (Consortium for Research on Educational Access, Transitions and Equity). It offered IPT daily for 9 months to eight mine-shaft clusters of gold miners at three mining companies over a 5 -year period. A further seven clusters were enrolled as control groups [28]. 27126 mine employees were enrolled in the study and of those $23644(87.8 \%)$, who had no evidence of active TB or contraindications, were started on IPT. These studies, although not changing the current paradigm of TB control, provide the opportunity to gain further insight into the potential contribution of TB prevention to gain the upper hand on the TB epidemic.

TB latency, testing and preventive treatment: what do we know and not know so far? Defining TB infection and latency

Infection with bacteria of the M. tuberculosis (MTB) complex is, in the overwhelming majority of cases acquired through the inhalation of bacilli-containing droplets. The antigen-specific response that is induced by infection results in the development of granuloma, which ideally provides evidence that a long-lasting protection against the development of $\mathrm{TB}$ disease has been activated. In this ideal scenario, where dormant bacilli, surrounded by the cells of the granuloma, greatly outnumber those in the replicative state, infection can be considered "latent". That condition is, clinically, characterised by the absence of any related disease [29]. Although persons with LTBI generally have a normal chest radiography image, subjects presenting radiological features suggestive of untreated and spontaneously healed TB (e.g. noncalcified apical fibrotic lesions), together with a history of prior exposure to contagious index cases, will also be considered to be "latently" infected carriers [30]. This operational distinction is somewhat artificial as, especially in children, the "latent" infection and primary disease are parts of a much less defined biological "continuum". Identification of LTBI is currently achieved, in the absence of a direct method, by immunological tests asserting that infection has occurred. Unfortunately they are not able to provide definitive evidence of the persisting presence of viable bacilli. Re-challenging the acquired immune response with mycobacterial antigens, using the in vitro interferon (IFN) $-\gamma$ release assays (IGRAs) or the in vivo tuberculin skin test (TST), may result in a positive test, suggesting infection. However, these tests provide discrimination neither between recent and older infections nor between subjects with LTBI and those suffering active disease [31].

A large number of prospective and retrospective studies have demonstrated that, without administration of antibiotics, $\sim 5 \%$ of immunocompetent persons with acquired LTBI can be expected to have their infections progressing to $\mathrm{TB}$ disease within 2 years of the infection. In practice, the 2-year risk-of-progression figure may even be higher among carefully preselected contacts with particularly intensive exposure [32]; however, it may also be lower [33].

Individual susceptibility factors may further increase the probability of acquiring MTB infection; these include active and passive smoking, young and advanced age, severe malnutrition, immune deficiency from any cause (mainly HIV-infection) including diabetes mellitus and immunosuppressive therapy, as well as some other medical conditions (e.g. chronic renal failure, silicosis, gastrectomy) (table 2) [2]. In addition, once infection has been acquired, multiple clinical and socio-epidemiological conditions may speed up progression from LTBI to TB disease [35-44].Thus, the risk of progression to TB disease among the estimated two billion individuals worldwide, who are infected with tuberculosis, may vary considerably. 
TABLE 2 Persons who should be given high priority for latent tuberculosis (TB)-infection treatment when found to be infected with TB

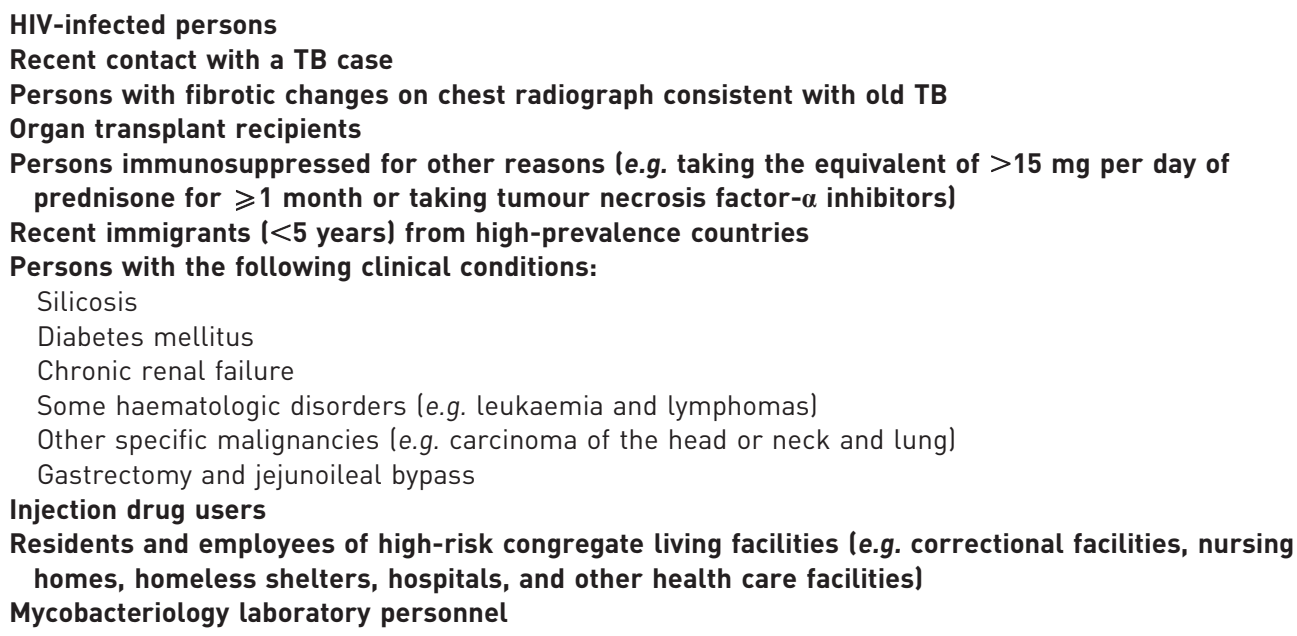

Reproduced and modified from [34].

\section{Sterilising $L T B I$}

Preventative treatment is a frequently used course of the anti-TB drug INH given for 6-9 months, aimed at sterilising latent infection. It can reduce life-time risk of progression to active disease and, thus, the risk of MTB transmission to further contacts [45]. Alternatively used regimens, such as INH plus rifampicin for 3 months, have proven to be comparably efficacious, but adverse effects may be more frequent than with INH monotherapy. Rifampicin monotherapy given to persons with INH-resistance or INH-intolerance for 4 months or in combination with INH for 3-4 months is well tolerated, but efficacy data are currently limited [table 3] [30, 45].

Hepatotoxic injuries, as the most important adverse effects of preventative treatment, are rare, but severe. These are not always predictable, but occur more frequently with older age, concomitant use of hepatotoxic medications and regular alcohol consumption [46]. Concern over this risk probably contributes to reduced acceptance of these regimes.

In fact, adverse effects experienced by IPT participants may vary depending on the number of investigated participants. The meta-analysis by STEELE et al. [47], pooled data from six very different studies on the incidence of hepatitis in adults, and provided a summary rate of clinical hepatitis of $210(0.6 \%)$ out of 38257 patients. In the Eastern Europe International Union Against Tuberculosis study [48], patients had a rate of INH-associated hepatotoxicity of $0.36 \%$ (6-month treatment) and of $0.44 \%$ (9-month treatment) based on related clinical symptoms. The early United States Public Health Service study [49] documented only 82 probable cases of INH hepatitis among 13838 receiving INH $(0.6 \%)$, and in the Thibela study on

\section{TABLE 3 Latent tuberculosis treatment regimens}

Drugs $\begin{gathered}\text { Duration } \\ \text { months }\end{gathered}$

\begin{tabular}{lccc}
\hline Isoniazid & 9 & Daily & 270 \\
& & Twice a week & 76 \\
Isoniazid & 6 & Daily & 180 \\
& & Twice a week & 52 \\
Isoniazid + rifampicin & $3-4$ & Daily & $90-120$ \\
Isoniazid + rifapentine & 3 & Once a week & 12 \\
Rifampicin & 4 & Daily & 120 \\
\hline
\end{tabular}

\#: directly observed therapy only. Data collated from [25, 26, 34, 45]. 
South African gold miners that included $>24000$ participants who started IPT, there were only 130 persons with adverse events $(0.5 \%)$ [50].

In contrast, some smaller studies, which have included treatment of HIV-infected persons, have reported grade 3 or 4 hepatitis in up to $5.3 \%$ in patients that were treated with INH [51]. In addition the recently published randomised study by STERLING et al. [25] shows a comparatively high proportion of hepatitis with $2.7 \%$ among $3745 \mathrm{INH}$ recipients.

Beside the new INH-RPT regimen given once a week for 3 months under DOT, other regimens may offer future advances in TB control, e.g. the combination of TMC207 (bedaquiline) and RPT given daily resulted in complete clearance of MTB in the mouse lung within 1 month [52]. A completely different approach would be to prevent the reactivation of LTBI by post-exposure vaccination, which is one of the targets of the ongoing research in developing new TB vaccines $[26,53,54]$. Currently, a total of 12 new vaccines are in development or that have already gone into clinical trials; thus the introduction of a new TB vaccine may be expected by the end of the decade [53].

\section{When and how to screen for LTBI?}

For more than a century, the only tool for detecting LTBI in a person with a history of exposure to an infectious TB case was the TST. However, commercially available IGRAs have emerged for the in vitro diagnosis of LTBI, and appear to be highly specific alternatives to the TST. These tests use antigens, coded by complex specific regions of difference (RD1 or RD11), that are absent from most non-tuberculous mycobacteria and from all strains of bacille Calmette-Guérin, to solicit secretion of IFN- $\gamma$ as a marker of immune responses by primed T-lymphocytes. This approach overcomes the cross-reactivity problems of the crude antigen mixture of purified protein derivative used in the TST.

When measuring IGRA and TST sensitivity against culture-confirmed TB cases, IGRAs seem to show significantly greater sensitivity in adults [55]. However, to date, this is not often in paucibacillary children $[56,57]$, where separate studies assessing IGRA performance are still limited. In the absence of a gold standard for LTBI, the "true" sensitivity of these tests cannot be assessed directly and the predominately sensitivity-driven negative predictive value for progression in the first years, following recent infection, is very high and nearly identical for both tests [58].

Induration size of the TST may be affected by HIV infection and other causes of immunosuppression (e.g. high-dose steroid use or haematological neoplastic disorders) showing a false-negative result, but this failing in sensitivity may also be the case when IGRA is used in such individuals, especially when the subjects have low CD4 cell counts [59].

Due to their higher specificity, IGRAs may help determine which people could actually benefit from preventative treatment, increasing pretest probability and thus reducing the number needed to treat (NNT) in order to prevent one TB case. Because screening with IGRAs requires only one visit to obtain and interpret results, IGRAs may also minimise loss of patients during follow-up. A disadvantage of the IGRAs is that blood samples must be processed within $8-30 \mathrm{~h}$ after collection, while white blood cells are still viable. Also, reversions of positive test results into negative results have been reported, which poses a challenge for interpreting a positive test result. [60]. Guidelines vary as to whether the more expensive IGRAs should be used as a primary or confirmatory test for identifying LTBI [61].

There is some evidence that commercial IGRAs are more reliable than the TST for predicting TB among high-risk persons. In the systematic review by RANGAKA et al. [62], it was reported that the incidence risk ratio was significant for the IGRA but not for the TST, showing more than a doubled risk for developing active TB given a positive IGRA, although in total a difference in the ability of IGRAs or TST to predict active TB was not confirmed. In another recently published meta-analysis study, the pooled positivepredictive value (PPV) for progression using commercial IGRAs was clearly higher, but overall still only moderate with a PPV of $6.8 \%$ for the IGRAs compared to $2.4 \%$ for the TST [56]. These analyses reveal the major limitations of current LTBI prevention strategies, namely that a high number of high-risk persons must still be treated to prevent a single TB case. Even when screening with a highly specific IGRA tool and subsequently treating those scored positive, assuming an 80\% IPT efficacy for the subgroup of "completercompliers", the NNT is not less than 12 individuals with LTBI [63]. As none of the currently available tests for LTBI (table 4), can accurately predict who will develop active TB among truly infected persons, the search for better diagnostic tools for LTBI must continue.

More advanced versions of the TST (e.g. the C-TST), focussing on the TB-specific early secretory antigen target -6 and the culture filtrate protein 10 , are needed in order to maximise test specificity. New IGRAs, based on novel antigens, are in the pipeline and their preliminary results have been published [64, 65]. 
TABLE 4 Approaches to diagnose latent tuberculosis (TB) Infection

\begin{tabular}{|c|c|c|c|c|c|c|c|}
\hline Assay & Modality & Antigen/s & Pathogenetic principle & $\begin{array}{l}\text { Diagnostic features and } \\
\text { progression to TB }\end{array}$ & $\begin{array}{c}\text { Visits } \\
n\end{array}$ & $\begin{array}{c}\text { Reading } \\
\mathrm{h}\end{array}$ & Diagnostic pitfalls \\
\hline TST & In vivo & $\begin{array}{l}\text { Tuberculin lcombination } \\
\text { of mycobacterial } \\
\text { proteins obtained from } \\
\text { the supernatant of } M \text {. } \\
\text { tuberculosis culture }\end{array}$ & $\begin{array}{l}\text { Identification of a cellular } \\
\text { immune response after } \\
\text { the intra-dermal } \\
\text { inoculation of tuberculin }\end{array}$ & $\begin{array}{l}\text { PPV } 0.024 \\
\text { NPV } 0.994\end{array}$ & 2 & $48-72$ & $\begin{array}{l}\text { Decreased sensitivity: } \\
\text { Immunocompromised individuals } \\
\text { Recent viral infections; } \\
\text { Recent } M \text {. Tuberculosis infection } \\
\text { Recent immunisation with live vaccines; } \\
\text { Individuals aged }<6 \text { months; } \\
\text { Inadequate management of tuberculin } \\
\text { Decreased specificity } \\
\text { BCG vaccination; } \\
\text { Exposure to non-tuberculous mycobacteria } \\
\text { Decreased positive predictive value } \\
\text { Low TB incidence areas. }\end{array}$ \\
\hline $\begin{array}{l}\text { IGRA } \\
\text { ELISA } \\
\text { ELISPOT }\end{array}$ & Ex vivo & $\begin{array}{l}\text { ESAT }-6 \\
\text { CFP-10 } \\
\text { TB7.7 }\end{array}$ & $\begin{array}{l}\text { Identification of the } \\
\text { production of IFN- } \gamma \text { after } \\
\text { exposure of lymphocytes } \\
\text { to mycobacterial antigens }\end{array}$ & $\begin{array}{l}\text { PPV } 0.068 \\
\text { NPV } 0.997\end{array}$ & 1 & $6-20$ & $\begin{array}{l}\text { Indeterminate result } \\
\text { No increase, in children; } \\
\text { No Increase, in immunocompromised } \\
\text { individuals }\end{array}$ \\
\hline
\end{tabular}

TST: tuberculin skin test; IGRA: interferon (IFN)- $\gamma$ release assay; ELISA: Enzyme-linked immunosorbent assay; M. tuberculosis: Mycobacterium tuberculosis; BCG: bacille CalmetteGuerin; ELISPOT: enzyme-linked immunosorbent spot assay; PPV: positive-predictive value; NPV: negative-predictive value ESAT-6: early secretory antigen target 6; CFP-10: culture filtrate protein 10.

\section{Indications for preventive treatment}

Regardless of the type of test used, LTBI treatment can only be considered effective and efficient as a programmatic approach when directed towards infected individuals bearing the highest risk of progression to disease. Especially in industrialised countries, where TB incidence has declined impressively over the past several decades, thanks to improved social conditions and the advancement in case detection and treatment, "targeted" testing and treatment for LTBI may be considered important cornerstones of the TB control strategy [65]. As the age-structured mathematical model of ABU-RADDAD et al. [66] focusing on the WHO Southeast Asia region suggests, TB incidence could be reduced dramatically by 2050 were LTBI treatment would be selectively expanded beyond low-incidence countries.

Of all American Thoracic Society(ATS)/CDC-defined groups with a high risk of developing TB, recently infected contacts of acid fast bacilli (AFB) smear-positive pulmonary TB source cases are most likely to benefit from LTBI treatment, as $\sim 20-30 \%$ of those have LTBI, and $1-3 \%$ have already progressed to TB disease at the date of tracing $[67,68]$. However, there is no unambiguous definition of a "close" contact, given the many possible modes of exposure to an infectious source. Selection of persons for screening generally begins with those contacts who share a household with the TB victim. This group may also include intimate contacts and frequent visitors to the home of the source, but a subtle approach to assessing the actual degree of proximity within a household contact is necessary to achieve a satisfactory pretest probability [69].

For practical purposes, in some recommendations [70, 71], a cumulative exposure time necessary for defining a "close" versus "casual" non-household contact has been stated, e.g. of $8 \mathrm{~h}$, if the index case is AFB smear-positive, or $40 \mathrm{~h}$, if only sputum culture-positive, but the duration and frequency of cough in the source case may outweigh the time factor for establishing contact categories (fig 1) [72].

A decision as to whether to expand the contact search in concentric circles should be made once testing of high-priority contacts is completed and once the extent of transmission of MTB in terms of the frequency of test conversion has been evaluated [69].

\section{LTBI in children}

Among close contacts, children and HIV-infected individuals require special attention. With their generally underdeveloped immune system, up to $40 \%$ of infants develop TB disease once infected and if untreated, and disease often develops faster than in adults [73]. Thus, formal investigation of juvenile contacts, especially of preschool children who are at the highest risk of progression [34], should proceed as quickly as possible to prevent the onset of serious, potentially life-threatening complications (e.g. TB meningitis). In high-prevalence countries the risk for MTB infection among children in contact with adult TB patients is $30-50 \%$, which is much higher than that reported for industrialised countries [74]. If not treated, these children will form a large pool for future disease. The current recommendations from WHO to treat preventively all children under the age of 5 years, as well as all HIV-infected children in contact with a case 


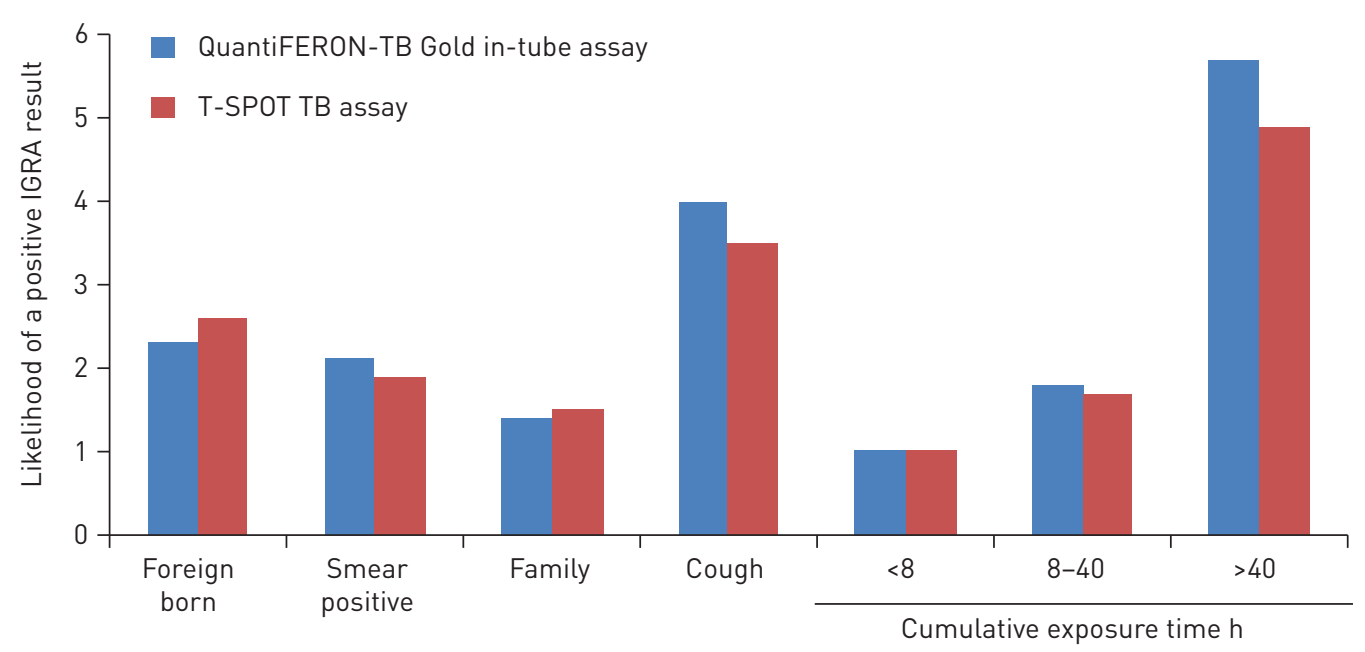

FIGURE 1 Association between positive interferon- $\gamma$ release assay results (IGRA) and foreign origin, acid-fast-bacilli smear positivity, family exposure source, coughing, and cumulative exposure time in contact persons. Reproduced and modified from [72] with permission from the publisher.

of smear-positive pulmonary $\mathrm{TB}$, with or without prior testing, is a reasonable and pragmatic approach and should be at least implemented in all settings where children have a high risk of exposure [75]. This pragmatic approach is not recommended in settings that are approaching the elimination phase.

\section{LTBI in HIV-infected persons}

In HIV-positive people with LTBI, the condition-induced failure of immune responses to restrict the growth of MTB accelerates reactivation, so that once infected with TB $\sim 30 \%$ of HIV patients will eventually contract active TB [76]. The annual risk of developing TB disease is 7-10\% [77].

Although WHO has actually reasserted the extensive implementation of IPT as one important component of TB control [78], global IPT coverage among HIV-infected persons is estimated to be at $<1 \%$ at the end of 2010 [79]. Among the 22 high-burden countries, South Africa has the highest number of HIV-coinfected TB cases with $60-80 \%$, thus generating a large pool of potentially infectious patients. In fact, there is evidence that transmission of TB is more likely attributable to the HIV co-infection than to HIV serotypenegative TB patients [80].

Combined antiretroviral therapy (ART) on its own is able to profoundly reduce TB incidence in HIVinfected patients in high- and low-burden countries [81], but independently ART, the efflcacy of 6 months of INH has been demonstrated also in HIV-infected patients with LTBI in numerous randomised clinical trials. In a recently published meta-analysis the effect of preventative treatment versus placebo was stronger for the prevention of active TB (RR 0.68, 95\% CI 0.54-0.85) in TST positive HIV-infected adults (RR 0.38, 95\% CI 0.25-0.57) compared to those who had a negative test or were untested (RR $0.89,95 \%$ CI $0.64-$ 1.24) [76]. However, one of the most frequently stated drawbacks to preventative treatment is that the protective duration of INH or of multiple-drug regimens (rifampicin with INH and/or pyrazinamide), is clearly shorter in HIV-infected persons than in non-HIV-infected persons and is often lost $\sim 1-2$ years after finishing treatment $[76,82]$.

This can be seen in the preliminary results of the Thibela TB study among gold miners in South Africa. Although TB incidence was reduced by $63 \%$ during the first 9 months after IPT, when compared to the miners who had not received IPT, the effect waned rapidly, and there was no significant protective effect of IPT beyond the 9 months. Consequently, TB incidence rates for the two groups, in the last 12 months of the study, were almost the same with 3.04 per 100 person-years in the intervention mines versus 2.96 in control mines [83].

However, there is general evidence that a clear benefit results from extending IPT in TST-positive, HIVinfected adults beyond the standard 6 months. In the BOTUSA (Botswana-USA Partnership) study, a 36month IPT course was well-tolerated with rates of adverse events similar to those in the placebo arm, and the NNT with prolonged IPT to prevent one case of TB and one death were 43 and 67 per year, respectively [84]. In contrast, administering 36-months of IPT to TST-negative patients was harmful by surprisingly tripling the risk of death as compared with 6-month IPT and, therefore, cannot be recommended in that 
subgroup. Thus the study points out that TST status, prior to IPT, is relevant and provides a strong rationale for targeting IPT according to TST status [85].

The additive benefit of combining INH and ART may further reduce TB risk in adult HIV-infected persons. Two cohort studies, Brazilian and South African HIV-infected adults who received ART or 6 months of IPT alone, have shown a reduction in TB risk of $40 \%$ to $60 \%$ when compared with patients not receiving any treatment, whereas IPT, followed by ART or both IPT with ART reduced TB rates by 80 to $90 \%$ [80, 86].

A further option may be administering post-treatment INH preventive treatment, which decreases the risk of recurrence by as much as 55\% in HIV-positive individuals with low CD4 cell counts. In the gold miners study by CHURCHYARD et al [87], the number of person-years of IPT required to prevent one case of recurrent TB among individuals with a CD4 cell count $<200 \cdot \mu \mathrm{L}^{-1}$, and $\geqslant 200$ cells $\cdot \mu \mathrm{L}^{-1}$ were 5 and 19 , respectively [87].

However, it should be kept in mind that these results are derived from studies from high TB incidence countries and, consequently, the conclusions of these studies cannot be "automatically" generalised countries with low TB incidence.

\section{LTBI in immigrants from high incidence regions}

A further important category of individuals who are, without preventative treatment, at a relatively high risk of infection and/or disease compared to the healthy autochthonous population are immigrants from countries with a high incidence of TB. Currently, in the USA and in many Western European countries, $>50 \%$ of persons who suffer from TB disease are young and foreign-born, whereas the disease affects mainly the elderly age groups in natives of the same countries $[5,88,89]$. The rate of TB in the foreign-born population in the UK is as much as 21 times higher than the rate for the UK-born population. Migrants often accumulate in metropolises, such as London, and a doubling of TB cases in London in the past decade has been attributed to immigration from high-prevalence countries [90].

In the USA, regulations require all immigrants to undergo TB screening in their countries of origin, and entering the USA is only permitted if sputum smears are negative in individuals with pulmonary infiltrates. However, $>80 \%$ of new immigrants to the USA who were subsequently diagnosed with active TB, of whom $50 \%$ received their positive diagnosis within 30 days after arrival, were screened within the 6 months prior to their arrival in the USA and had had a negative result $[88,91]$. Accordingly, the ability of current "overseas" screening programmes to detect TB based on chest radiography and AFB is considered to be low, leaving persons with AFB-negative active $\mathrm{TB}$, who constitute the majority (65.1\%) of new immigrants to the USA, undetected [92].

Screening for LTBI in immigrants to low-burden countries is important because most of the TB cases in this group seem to arise through the reactivation of infections acquired abroad. Although the still valid USA guidelines recommend screening and treatment solely for foreign-born persons living in the USA for $\leqslant 5$ years [34], nearly half of all TB cases in the USA occur in foreign-born persons who have been in the USA for $>5$ years, and most of those cases are due to the reactivation of a latent infection [93]. One fifth of recent immigrants to the Netherlands were diagnosed as having LTBI by a positive IGRA result and of those $\sim 10 \%$ developed TB disease within 2 years of their arrival [94].

Since 2006 in the UK, the National Institute for Health and Clinical Excellence (NICE) guidelines for new entrant TB screening recommend chest radiography for immigrants from countries with a TB incidence $>40$ per $100000[95,96]$. In the case of normal chest radiography results, TST should subsequently be performed followed by an IGRA for those with positive a TST, to confirm the diagnosis of LTBI. An IGRA first protocol followed by chest radiography has been suggested to detect more LTBI cases and to be more cost-effective than the NICE guidelines for screening new entrants from countries with TB incidence $>150$ per 100000 [57] or $>200$ per 100000 [97]. Stratification of immigrants by country of origin and status of immigration may further increase the effectiveness of LTBI treatment [98-100].

\section{LTBIs in patients with diabetes mellitus}

Another, still underestimated condition that may contribute to reactivation from LTBI is diabetes mellitus. Diabetes mellitus is long known to be a medical condition that impairs immunity to MTB but has, to date, received little attention. Irrespective of the chosen study design, diabetes mellitus is generally associated with a two-to-threefold increased risk of both pulmonary and extrapulmonary TB [101-103].

Based on the assumption that the relative risk of developing TB is clearly increased in diabetics, but that the absolute risk of TB appears to be generally low, recommendations for actively screening of diabetics for LTBI are, to date, sparse; however, screenings are included in the USA guidelines [104]. Former NICE guidelines [105] even explicitly stated that there is no need to educate diabetes patients about symptoms 
suggestive of TB disease (section 10.2.4) although that would conceivably help to minimise delay in starting the respective diagnostic procedures.

In fact, the rapidly growing pool of patients in the ongoing diabetes mellitus pandemic increases the fraction of TB cases attributable to diabetes mellitus [106], and so LTBI in diabetics is becoming a global issue for the TB community.

Estimates for India, where there were an estimated 20.7 million adults with diabetes mellitus and 900000 incidents of adult-pulmonary TB in the year 2000, suggests that diabetes mellitus accounts for $\sim 15 \%$ of pulmonary TB and even $20 \%$ of AFB smear-positive cases [107]. It was found that around one-third of Asians with newly-diagnosed TB in England have diabetes mellitus, and it has been calculated that $\sim 11 \%$ of the new cases of pulmonary TB that occurred in England in 2005 were triggered by diabetes mellitus [108].

Thus, a decision on whether diabetics, especially in populations with an expected high prevalence of LTBI, such as immigrants, should be regularly screened for pre-existing LTBI is overdue.

\section{LTBI in patients with immunosuppressive therapy}

There exist further immunosuppressive medical conditions for which an LTBI control strategy appears to be indicated, a strategy, however, for which experts still struggle to find consensus. Post-transplant TB, has been reported to be a rare complication after solid organ or haematopoietic stem-cell transplantation with a 20-74-fold and two-fold TB risk, respectively [109]. In recipients with at least one additional risk factor for MTB infection, LTBI screening should be carried out before the administration of immunosuppressive medication. A general decrease in test sensitivity of both TST and IGRAs has been demonstrated when immunosuppression increases. However, to date there is insufficient evidence to demonstrate whether treatment should start before or after transplantation and to guide optimal duration of preventative treatment [109].

In LTBI patients treated with newer tumour necrosis factor (TNF)- $\alpha$ inhibitors for various chronic inflammatory and autoimmune diseases, the formation of protective granuloma may be weakened at the site of mycobacterial infection, for which the key pro-inflammatory cytokine TNF is required as an essential component of host immunity to keep mycobacteria in their latent phase [110].

Since the introduction of TNF- $\alpha$ antagonist, an increase in the cases of TB within the treated population has been observed. In the USA, TB rates have been reported at 144 per 100000 in patients who are receiving treatment with infliximab and 35 per 100000 in those who receive etanercept [110], versus 6.2 per 100000 in the general population.

As with patients awaiting transplants, screening for LTBI should be performed before initiating TNF- $\alpha$ inhibitor therapy. Strategies to start preventive treatment with INH 4 weeks prior to treatment with TNF- $\alpha$ inhibitors and to continue INH preventative treatment during treatment with TNF- $\alpha$ inhibitors for usually a total of 9 months, have shown to decrease the TB rate by 78\% [111]. Nevertheless, apart from mandatory chest radiography examination and the compiling of complete medical history, the recommendations that tests should be used to detect LTBI are highly heterogeneous. Because the TST may be falsely positive, in some low-incidence countries (e.g. Germany, France, Austria and Switzerland) it is recommended only for exceptional situations, the IGRA being the test of first choice. Whilst also in IGRA testing false-negative results are fairly common, the other low-incidence countries recommend the use of either the TST or the IGRA, the use of both tests simultaneously or alternatively a dual step approach [61]. This heterogeneity points to a need for further, more conclusive, comparative longitudinal studies to estimate the risk for progression to TB after IGRA-based and/or TST-based diagnosis of LTBI in those patients starting therapy with TNF antagonists.

\section{Cost-effectiveness of preventive programmes for LTBI}

Cost-effectiveness analyses are an important component of TB control-programme delivery decision making. Although there is growing numbers of such economic analyses, their results cannot be not be generalised. Beside apparent differences in costs of tests and drugs recommended for treating, they also depend on estimates of test accuracy, the socio-environmental conditions of high-risk groups in the respective countries, vaccination coverage or protective effect and effectiveness of and compliance to preventative treatment. Interventions that produce a quality adjusted life year (QALY) for $\leqslant \$ 50,000$ are often considered in the literature as bargains, whilst those requiring $\geqslant \$ 100,000$ per QALY are considered out of the question [112]. Although the results of several economic analyses are predominately driven by divergent estimates of sensitivity and specificity of TST versus IGRA in the absence of an ultimate "gold standard", in a larger number of studies with different economic models used, LTBI treatment, following 
positive test results has been shown to be less costly than "doing nothing" in many settings, regardless of whether TST or IGRA is used [113]. However, a prerequisite for economic benefits is a high prevalence of LTBI and/or a high risk of developing active TB in the particular target population.

In their recently published work LINAS et al. [93] emphasised the varying advantages of both tests by analysing the cost-effectiveness of LTBI treatment for a bundle of high-risk groups under real-life USA conditions. In their comprehensive decision model they estimated adherence to a planned 9-month INH course of only $24-58 \%$ dependent on the various risk groups, an assumed IGRA sensitivity of $6 \%$ lower than that assumed for the TST ( $83 \%$ versus $89 \%$ ) in all risk groups and a specificity only $7 \%$ lower for the TST in foreign-born, compared to the respective figure of $99 \%$ for IGRA.

These studies concluded that screening with either TST or IGRA was likely to be cost-effective across all plausible estimates in close contacts (all age groups), HIV-positives (both risk groups $\leqslant \$ 50,000$ threshold), and recent immigrants and foreign-born persons aged $<45$ years, who had resided in the USA for $>5$ years. Interestingly, either due to an assumed low LTBI prevalence (e.g. in patients taking immunosuppressive drugs) or extraordinarily poor adherence to INH (homeless and i.v. drug abusers) treatment of all the other currently recommended TB risk groups were, in general, too costly, only falling below the $\$ 100,000$ threshold when rates of reactivation of $11 \%$ per 100 person-years were assumed and TST screening had been performed.

In general, DOT may further improve the effectiveness of PT, especially in risk groups such as drug users, and may not lead to additional cost because they are outweighed if DOT provides a $10 \%$ increment in adherence over self-administered PT [114].

\section{LTBI in contacts of patients with MDR-TB}

Given appropriate and evidence-based future options, screening and preventive treatment of contacts of patients with MDR-TB may be even more cost-effective, considering the high cost of treatment of MDR-TB and the often unfavourable prognosis of MDR-TB once the disease is diagnosed. BECERRA et al. [115] demonstrated in Peru that $91 \%$ of the 142 household contacts of MDR-/extremely drug-resistant (XDR)-TB cases with active TB who underwent drug susceptibility testing also had MDR-TB [115].

In two recently published studies the mean costs for drug-susceptible TB in Germany were $€ 7364$ in adults, but amounted to $€ 52259$, more than sevenfold higher, for treatment of MDR-TB [116]. The cost per MDR-TB patient treated in 2010 would have been \$20 910 in Estonia and \$22 512 in the Russian Federation [117].

However, there is no consensus on the management of contacts of MDR-TB patients [118] and very little evidence on what preventative treatment provides the best treatment outcomes of infected contacts of MDR-TB patients $[119,120]$. The ATS/CDC joint statement pragmatically recommends regimens including two drugs to which the source case's TB strain is susceptible [34], whereas previously proposed standard regimens include combinations of pyrazinamide, ethambutol and a fluoroquinolone [121]. The National Department of Health in South Africa [119] suggests giving high-dose INH $\left(15 \mathrm{mg} \cdot \mathrm{kg}^{-1}\right)$ to asymptomatic contacts aged $\leqslant 5$ years, pre-supposing that treatment of drug-susceptible strains, as well as strains with low-level resistance (a mutation in the mycobacterial inhA promoter region), would be effective [122]. 105 infected adult and child contacts of 19 MDR-TB source cases in Chuuk (Federated States of Micronesia) were given a 12-month course of fluoroquinolone alone or in combination with ethionamide for one of two identified strains, and fluoroquinolone with ethambutol for the other in a DOT programme. None of the 93 patients who completed treatment had, at the time of the report, developed TB, but due to lack of statistical power no conclusions could be drawn [123].

To date no randomised controlled trials have been published on the efficacy of possible treatment combinations for latent MDR-TB infection [124], and thus the old principle "intention-to-test is intentionto-treat" is clearly not applicable to MDR-TB contacts. Accordingly, the most recent European Centre for Disease Prevention and Control (ECDC) guidance [123] provides two options for the management of contacts of MDR-TB and XDR-TB patients. 1) Preventive therapy, where the selection of the drugs should be based on the drug susceptibility pattern of the source case's likely infecting strain; local patterns of drug resistance; and the potential adverse events in individual patients, taking into account age and other risk factors. 2) Follow-up with careful clinical observation of the identified contact considered to have LTBI. The European Union Standards for Tuberculosis Care (ESTC) [125] also suggest close clinical monitoring solely, but stresses the necessity for detailed information and close follow-up of infected contacts if no preventative treatment is prescribed. Therefore, further studies are urgently needed for achieving effective treatment schedules. 
Furthermore, it is useful to underline that prevention of active TB begins with the prevention of TB infection. One important practice, especially in high-prevalence settings, is the reduction in emission of infectious aerosols through the wearing of surgical face masks by infectious patients, once other interventions (awareness, early access and diagnosis in the community) have been ensured. Using a quantitative biological air-sampling and transmission model in which susceptible guinea pigs served as surrogate hosts, easy-to-use-surgical masks worn by patients in a South African ward reduced the risk of MDR-TB transmission by up to $56 \%$ [126].

\section{Accelerating towards zero infections, zero cases, zero deaths: playing the preventive treatment card}

In conclusion, there are a variety of options in which preventative treatment may help to achieve shrinkage of the worldwide pool of latent infections. With emphatic determination to achieve the United Nations Millennium Development Goal of a 50\% reduction in the global prevalence and mortality of TB by the year 2015 [127], current TB control programmes still focus almost solely on the concept of reducing primary transmission of $\mathrm{MTB}$ by prompt case-finding and adequate treatment of infectious patients. Beyond that, though, to achieve the highly ambitious goal of TB elimination by 2050, it will be necessary to combine, with the same determination, the transmission-blocking approach with

\section{TABLE 5 Core activities to achieve tuberculosis (TB) elimination in Europe}

TB elimination area
Surveillance

Laboratory services

Prompt and quality TB care for all

\section{Multidrug-resistant/extensively drug-resistant TB and TB/HIV co-infection}

\section{New tools for TB control and elimination}

\author{
Partnership and collaboration \\ with countries
}

\section{Core activities}

Maintaining and enhancing government commitment to engage with the nonstate, private sector and sustain TB control and elimination

Planning and retaining the adequate human resources necessary to ensure quality TB control and elimination activities at all levels

Establishing targets, implementing quality surveillance and monitoring lincluding transition patterns with molecular epidemiologyl and adequate modelling to evaluate impact

Improving laboratory services for quality microbiology diagnosis and implementation of ongoing transmission and molecular epidemiology studies

Identifying and addressing social- and economical-TB determinants (e.g. housing, nutrition, smoking, etc.) with particular attention to high-risk groups and vulnerable populations to reduce incidence

Improving efficiency of services and ensuring financing of services through social protection mechanisms and universal health coverage/free care

Improving service deliveries ensuring early diagnosis, efficient treatment and through-care of all types of TB

Preventing emerging of further drug resistance and effective treatment of existing multidrug-resistant/extensively drug-resistant TB cases

Preventing further spread of HIV infection, effective testing and anti-retroviral treatment through quality implementation of TB/ HIV collaborating activities

Ensuring preventive treatment of latent TB infection and airborne infection control

Promoting innovative research on new diagnostics, drugs and vaccines

Targeting adoption of the new tools to accelerate achievement of TB elimination with special attention to children, people infected with HIV and other immunosuppressed individuals (e.g. tumour necrosis factor- $\alpha$ ).

Ensuring rational use of new drugs for the treatment of TB and latent TB infection

Developing joint planning and monitoring and evaluation, and pan-European collaboration to sustain TB elimination 
well-resourced, targeted LTBI treatment strategies to ensure adequate protection of the already identified vulnerable risk-groups [128].

As a first step in this direction, the current ESTC recommendations go beyond the traditional focus on close contacts to include preventative treatment for persons with immunosuppressive co-morbidities such as diabetes mellitus, and those under immunosuppressive therapy, all of which increase the risk of mycobacterial reactivation.

The ESTC jointly developed by the ECDC and the ERS, demonstrate that TB (which continues to be a significant European public health threat) needs to be tackled with European specific recommendations $[125,129]$.

In response to the alarming occurrence of MDR-/XDR-TB cases in the WHO European Region, a consolidated action plan has been developed for 2011-2015 for all 53 Member States of the WHO European Region and partner states [130]. According to the standards established by that plan, preventative treatment against TB development may be relevant to the completion of the efforts to reduce the burden of disease, in addition to the establishment of improved and permanent TB-control programmes based on case finding and treatment.

Despite its inclusion in the consolidated action plan, preventative treatment is currently not largely implemented in most National Tuberculosis Control Programmes (NTPs), and the question of which riskgroups should be addressed for such treatment remains a matter of significant controversy. In line with the England and Wales TB Programme [131] the US CDCs TB elimination-focussed NTP places equal emphasis on the practice of preventative treatment and the completion of treatment against active disease, and claims improving treatment initiation for contacts of AFB sputum smear-positive patients to $88 \%$ and treatment completion rates of $79 \%$ [132]. On the contrary, in the NTPs of other large priority countries, such as India, China or Russia, preventative treatment is not even mentioned [133]. Other NTPs such as of South Africa recommend treatment only in TST-positive children and HIV-infected people, but in such resource-limited settings NTPs often lack adequate funding to ensure chest radiography is performed to exclude active TB and to procure tuberculin for TST. Many NTP managers, too, fear poor adherence to long IPT regime, sideeffects, or the development of resistance when active TB was wrongfully excluded [134]. As a consequence, even the contacts at highest risk of developing TB may not be managed adequately, and easy opportunities for prevention are missed [135].

\section{Conclusions}

Three core components are necessary to implement the elimination strategy fully: 1) improve diagnosis of LTBI; 2) improve regimens to treat LTBI; and 3) ensure public health commitment to make 1) and 2) possible.

New biomarkers that could identify those at higher risk of developing TB would greatly facilitate precise targeting of those persons who should undergo preventative treatment, by discriminating them from MTBinfected subjects with chronic "stable" infection and those who may already have cleared viable MTB strains [136]. In this context, the aim to achieve sterile eradication of MTB from the latently infected host may become an additional approach in the future $[53,54]$.

New drugs are presently facing the treatment arena, including newly developed compounds (e.g. bedaquiline, PA-824, delamanid) and existing drugs with new indications (e.g. linezolid, meropenem and co-trimoxazole) [17-20, 137-139]. On top of adequate public health attention aimed at protecting these new drugs from irrational use $[11,140,141]$, research on how to include these new molecules into new, effective and shorter new LTBI regimens (also able to sterilise MDR-TB strains) facilitating adherence need to be intensified involving producers, regulatory authorities and national programmes under the WHO's coordination.

Last but not least sound policies need to be implemented at the national level in Europe to ensure that individuals with LTBI at high risk for development of TB are diagnosed and treated, and that the whole package of core interventions necessary to reach elimination are duly activated (table 5) [142].

\section{Acknowledgements}

The European Forum for TB Innovation members and observers, in alphabetic order, are as follows: A. Khan (Pakistan), F. Blasi (Italy), S. Borisov (Russian Federation), J. Caminero (Spain), L. Cuevas (United Kingdom), M. Danilovits (Estonia), M. Dara (Observer, Denmark), A. Detjen (USA), F. Drobniewski (UK), C. Erkens (The Netherlands), P. Gondrie (The Netherlands), E. Jaramillo (Observer, Switzerland), C. Lange (Germany), R. Loddenkemper (Germany), D. Maher (Observer, Switzerland), GB. Migliori (Italy), T. Mori (Japan), A. Mosneaga (Republic of Moldova), L.B. Reichmann (USA), P. Thorn (UK), C. Yu (Invited guest, Philippines), J-P. Zellweger (Switzerland). The opinion of the members and observers of the forum represents their individual opinion and in no way should be interpreted as institutional endorsement of the ideas and concepts hereby expressed. 


\section{References}

1 World Health Organization. Tuberculosis Fact Sheet No 104. Available from: http://www.who.int/mediacentre/ factsheets/fs104/en/index.html Date last accessed: Janaury 16, 2013; Date last updated: February 2013.

2 Horsburgh CR Jr, Rubin EJ. Clinical practise. Latent Tuberculosis Infection in the United States. N Engl J Med 2011; 364: 1441-1448.

3 Raviglione M, Marais B, Floyd $\mathrm{K}$, et al. Scaling up interventions to achieve global tuberculosis control: progress and new developments. Lancet 2012; 379: 1902-1913.

4 Onozaki I, Raviglione MC. Stopping tuberculosis in the 21st century: Goals and strategies. Respirology 2010; 15: 32-43.

5 Broekmans JF, Migliori GB, Rieder HL, et al. European framework for tuberculosis control and elimination in countries with a low incidence. Eur Respir J 2002; 19: 765-775.

6 Raviglione MC, Uplekar MW. WHO’s new Stop TB Strategy. Lancet 2006; 367: 952-955.

7 Tadolini M, Migliori GB. The WHO strategy for tuberculosis control and elimination. In: Lange C, Migliori GB, ed. Tuberculosis. Eur Respir Monogr 2012; 58: 242-253.

8 Tadolini M, Centis R, D'Ambrosio L, et al. TB and MDR-TB: what is new in 2012? Breathe 2012; 9: 101-111.

9 World Bank. World Development Report 1993. Investing in Health. New York. Oxford University Press. 1993; pp. 63.

10 Raviglione MC, Pio A. Evolution of WHO policies for tuberculosis control, 1948-2001. Lancet 2002; 359: 775-780.

11 D’Ambrosio L, Centis R, Migliori GB. Multidrug-Resistant Tuberculosis. N Engl J Med 2012; 367: 2154.

12 World Health Organization. Report of the 12th Meeting WHO Strategic and Technical Advisory Group for Tuberculosis (STAG-TB) 18-20 June 2012. Document WHO/HTM/TB/2012.7. Geneva, World Health Organization, 2012.

13 Keshavjee S, Farmer PE. TB, drug resistance, and the history of modern medicine. $N$ Eng J Med 2012; 367: 931-936.

14 Clancy L, Rieder HL, Enarson DA, et al. Tuberculosis elimination in the countries of Europe and other industrialized countries. Eur Respir J 1991; 4: 1288-1295.

15 Veen J, Migliori GB, Raviglione M, et al. Harmonisation of TB control in the WHO European region: the history of the Wolfheze Workshops. Eur Respir J 2011; 37: 950-959.

16 Drobniewski F, Nikolayevskyy V, Balabanova Y, et al. Diagnosis of tuberculosis and drug resistance: what can new tools bring us? Int J Tuberc Lung Dis 2012; 16: 860-870.

17 Gler MT, Skripconoka V, Sanchez-Garavito E, et al. Delamanid for multidrug-resistant pulmonary tuberculosis. N Engl J Med 2012; 366: 2151-2160.

18 Skripconoka V, Danilovits M, Pehme L, et al. Delamanid improves outcomes and reduces mortality for multidrugresistant tuberculosis. Eur Respir J 2013; 41: 1393-1400.

19 Diacon AH, Dawson R, von Groote-Bidlingmaier F, et al. 14-day bactericidal activity of PA-824, bedaquiline, pyrazinamide and moxifloxacin combinations: a randomised trial. Lancet 2012; 380: 986-983.

20 Migliori GB, Sotgiu G. Treatment of tuberculosis: have we turned the corner? Lancet 2012; 380: 955-957.

21 Blasi F, Reichman LB, Migliori GB. Presenting the European Forum for TB Innovation: innovative thinking in progressing towards TB elimination in Europe. Eur Respir J 2012; 40: 806-808.

22 Zorini AO. On a new method of antituberculosis chemioprophylaxis employing isonicotinic acid hydrazide. Sci Med Ital 1957; 6: 37-47.

23 Hanson ML, Comstock GW, Haley CE. Community isoniazid prophylaxis program in an underdeveloped area of Alaska. Public Health Rep 1967; 82: 1045-1056.

24 Ferebee SH. Controlled chemoprophylaxis trials in tuberculosis. A general review. Bibl Tuberc 1970; 26: 28-106.

25 Sterling TR, Villarino ME, Borisov AS, et al. Three months of rifapentine and isoniazid for latent tuberculosis infection. N Engl J Med 2011; 365: 2155-2166.

26 Centers for Disease Control and Prevention (CDC). Recommendations for use of an isoniazid-rifapentine regimen with direct observation to treat latent Mycobacterium tuberculosis infection. MMWR Morb Mortal Wkly Rep 2011; 60: 1650-1653.

27 Hanifa Y, Grant AD, Lewis J, et al. Prevalence of latent tuberculosis infection among gold miners in South Africa. Int J Tuberc Lung Dis 2009; 13: 39-46.

28 Smart T. Thibela TB: community-wide IPT did not improve TB control in South Africa's mines at the population level. nam aidsmap. www.aidsmap.com/Thibela-TB-Community-wide-IPT-did-not-improve-TB-control-inSouth-Africas-mines-at-the-population-level/page/2285760/ Date last accessed: March 2012.

29 Esmail H, Barry CE 3rd., Wilkinson RJ. Understanding latent tuberculosis: the key to improved diagnostic and novel treatment strategies. Drug Discov Today 2012; 17: 514-521.

30 Menzies D, Al Jahdali $\mathrm{H}, \mathrm{Al}$ Otaibi B. Recent developments in treatment of latent tuberculosis infection. Indian J Med Res 2011; 133: 257-266.

31 Mack U, Migliori GB, Sester M, et al. LTBI: latent tuberculosis infection or lasting immune responses to $M$. tuberculosis? A TBNET consensus statement. Eur Respir J 2009; 33: 956-973.

32 Diel R, Loddenkemper R, Niemann S, et al. Negative and positive predictive value of a whole-blood interferon- $\gamma$ release assay for developing active tuberculosis: an update. Am J Respir Crit Care Med 2011; 183: 88-95.

33 Kik SV, Franken WP, Mensen M, et al. Predictive value for progression to tuberculosis by IGRA and TST in immigrant contacts. Eur Respir J 2010; 35: 1346-1353.

34 American Thoracic Society. Targeted tuberculin testing and treatment of latent tuberculosis infection. Am J Respir Crit Care Med 2000; 161: Suppl. 3, S221-S247.

35 Ferrara G, Murray M, Winthrop K, et al. Risk factors associated with pulmonary tuberculosis: smoking, diabetes and anti-TNF $\alpha$ drugs. Curr Opin Pulm Med 2012; 18: 233-240.

36 Den Boon S, Verver S, Marais BJ, et al. Association between passive smoking and infection with Mycobacterium tuberculosis in children. Pediatrics 2007; 119: 734-739.

37 Creswell J, Raviglione M, Ottmani S, et al. Tuberculosis and noncommunicable diseases: neglected links and missed opportunities. Eur Respir J 2011; 37: 1269-1282. 
ecological study. Eur Respir J 2012; 40: 925-930. Lönnroth K, Jaramillo E, Williams BG, et al. Drivers of tuberculosis epidemics: The role of risk factors and social Lin H, Ezzati M, Murray M. Tobacco smoke, indoor air pollution and tuberculosis: a systematic review and metaanalysis. PLoS Med 2007; 4: e20.

41 Jeon CY, Murray MB. Diabetes mellitus increases the risk of active tuberculosis: a systematic review of 13 observational studies. PLoS Med 2008; 5: e152.

42 Lönnroth K, Williams BG, Cegielski P, et al. A consistent log-linear relationship between tuberculosis incidence and body mass index. Int J Epidemiology 2010; 9: 149-155.

43 Lönnroth K, Williams BG, Stadlin S, et al. Alcohol use as a risk factor for tuberculosis - a systematic review. BMC Public Health 2008; 8: 289.

44 Lönnroth K, Castro K, Chakaya JM, et al. Tuberculosis control and elimination 2010-50: cure, care and social change. Lancet 2010; 375: 1814-1829.

45 Lobue P, Menzies D. Treatment of latent tuberculosis infection: An update. Respirology 2010; 15: 603-622.

46 Landry J, Menzies D. Preventive chemotherapy. Where has it got us? Where to go next? Int J Tuberc Lung Dis 2008; 12: $1352-1364$.

47 Steele MA, Burk RF, DesPrez RM. Toxic hepatitis with isoniazid and rifampin. A meta-analysis. Chest 1991; 99 : 465-471.

48 Efficacy of various durations of isoniazid preventive therapy for tuberculosis: Five years of follow-up in the IUAT trial. Bull World Health Organ 1982; 60: 555-564.

49 Kopanoff DE, Snider DE Jr, Caras GJ. Isoniazid-related hepatitis: a U.S. Public Health Service cooperative surveillance study. Am Rev Respir Dis 1978; 117: 991-1001.

50 Grant AD, Mngadi KT, van Halsema CLet al. Adverse events with isoniazid preventive therapy: experience from a large trial. AIDS 2010; 24: Suppl. 5, S29-S36.

51 Hawken MP, Meme HK, Elliott LC, et al. Isoniazid preventive therapy for tuberculosis in HIV-1-infected adults: results of a randomized controlled trial. AIDS 1997; 11: 875-882.

52 Zhang T, Li SY, Williams KN, et al. Short-course chemotherapy with TMC207 and rifapentine in a murine model of LTBI. Am J Respir Crit Care Med 2011; 184: 732-737.

53 Ottenhoff THM Kaufmann SHE Vaccines against tuberculosis. where are we and where do we need to go? PLoS Pathog 2012; 8: e1002607.

54 Brennan MJ, Stone MR, Evans T. A rational vaccine pipeline for tuberculosis. Int J Tuberc Lung Dis 2012; 16: $1566-1573$.

55 Sester M, Sotgiu G, Lange C, et al. Interferon- $\gamma$ release assays for the diagnosis of active tuberculosis: a systematic review and meta-analysis. Eur Respir J 2011; 37: 100-111.

56 Machingaidze S, Wiysonge CS, Gonzalez-Angulo Y, et al. The utility of an interferon- $\gamma$ release assay for diagnosis of latent tuberculosis infection and disease in children: a systematic review and meta-analysis. Pediatr Infect Dis J 2011; 30: 694-700.

57 Mandalakas AM, Detjen AK, Hesseling AC, et al. Interferon- $\gamma$ release assays and childhood tuberculosis: systematic review and meta-analysis. Int J Tuberc Lung Dis 2011; 15: 1018-32.

58 Diel R, Loddenkemper R, Nienhaus A. Predictive value of interferon- $\gamma$ release assays and tuberculin skin testing for predicting progression from latent TB infection to disease state: a meta-analysis. Chest 2012; 142; 63-75.

59 Cattamanchi A, Smith R, Steingart KR, et al. Interferon- $\gamma$ release assays for the diagnosis of latent tuberculosis infection in HIV-infected individuals: a systematic review and meta-analysis. J Acquir Immune Defic Syndr 2011; 56: 230-238.

60 Van Zyl-Smit RN, Zwerling A, Dheda K, et al. Within-subject variability of interferon-g assay results for tuberculosis and boosting effect of tuberculin skin testing: a systematic review. PLoS One 2009; 4: e8517.

61 Denkinger CM, Dheda K, Pai M. Guidelines on interferon- $\gamma$ release assays for tuberculosis infection: concordance, discordance or confusion? Clin Microbiol Infect 2011; 17: 806-814.

62 Rangaka MX, Wilkinson KA, Glynn JR, et al. Predictive value of interferon- $\gamma$ release assays for incident active tuberculosis: a systematic review and meta-analysis. Lancet Infect Dis 2012; 12: 45-55.

63 Diel R, Wrighton-Smith P, Zellweger JP. Cost-effectiveness of interferon- $\gamma$ release assay testing for the treatment of latent tuberculosis. Eur Respir J 2007; 30: 321-332.

64 Casal C, Bezos J, Díez-Guerrier A, et al. Evaluation of two cocktails containing ESAT-6, CFP-10 and Rv-3615c in the intradermal test and the interferon $\gamma$ assay for diagnosis of bovine tuberculosis. Prev Vet Med 2012; 105: 149-154.

65 Whitworth HS, Scott M, Connell DW, et al. IGRAs - the gateway to T cell based TB diagnosis. Methods 2013; 61: 52-62.

66 Abu-Raddad LJ, Sabatelli L, Achterberg JT, et al. Epidemiological benefits of more-effective tuberculosis vaccines, drugs, and diagnostics. Proc Natl Acad Sci USA 2009; 106: 13980-13985.

67 Morrison J, Pai M, Hopewell PC. Tuberculosis and latent tuberculosis infection in close contacts of people with pulmonary tuberculosis in low-income and middle-income countries: a systematic review and meta-analysis. Lancet Infect Dis 2008; 8: 359-368.

68 Reichler MR, Reves R, Bur S, et al. Evaluation of investigations conducted to detect and prevent transmission of tuberculosis. JAMA 2002; 287: 991-995.

69 Erkens CGM, Kamphorst M, Abubakar I, et al. Tuberculosis contact investigation in low prevalence countries: a European consensus. Eur Respir J 2010; 36: 925-949.

70 Diel R, Loytved G, Nienhaus A, et al. Neue empfehlungen fur die umgebungsuntersuchungen bei tuberkulose. [New recommendations for contact tracing in tuberculosis.]. Pneumologie 2011; 65: 359-378.

71 HandbuchTuberkulose 2011. Available from: www.tbinfo.ch/uploads/media/Handbuch_Tuberkulose_2012_de.pdf Date last accessed: Date last updated: April 2012. Date last accessed: June 27, 2013.

72 Diel R, Loddenkemper R, Meywald-Walter K, et al. Comparative performance of tuberculin skin test, QuantiFERON-TB-Gold In Tube assay, and T-Spot.TB test in contact investigations for tuberculosis. Chest 2009; 135: 1010-1018. 
73 Shingadia D, Novelli V. Diagnosis and treatment of tuberculosis in children. Lancet Infect Dis 2003; 3: $624-632$.

74 Nakaoka H, Lawson L, Squire SB, et al. Risk for tuberculosis among children. Emerg Infect Dis 2006; 12: 1383-1388.

75 World Health Organization. Guidance for National Tuberculosis Programmes on the Management of Tuberculosis in Children.Document WHO/HTM/TB/2006.371. Geneva, World Health Organization, 2006.

76 Akolo C, Adetifa I, Shepperd S, et al. Treatment of latent tuberculosis infection in HIV infected persons. Cochrane Database Syst Rev 2010; 1: CD000171.

77 Selwyn PA, Hartel D, Lewis VA, et al. A prospective study of the risk of tuberculosis among intravenous drug users with human immunodeflciency virus infection. N Eng J Med 1989; 320: 545-550.

78 World Health Organization Department of HIV/AIDS and Stop TB Department. Guidelines for intensified tuberculosis case-finding and isoniazid preventive therapy for people living with HIV in resource constrained settings. Geneva, World Health Organization, 2011.

79 Boyles TH, Maartens G. Should tuberculin skin testing be a prerequisite to prolonged IPT for HIV-infected adults? Int J Tuberc Lung Dis 2012; 16: 857-859.

80 Martinson NA, Hoffmann CJ, Chaisson RE. Epidemiology of tuberculosis and HIV: recent advances in understanding and responses. Proc Am Thorac Soc 2011; 8: 288-293.

81 Girardi E, Palmieri F, Angeletti C, et al. Impact of previous ART and of ART initiation on outcome of HIVassociated tuberculosis. Clin Dev Immunol 2012; 2012: 931325.

82 Johnson JL, Okwera A, Hom DL, et al. Duration of efflcacy of treatment of latent tuberculosis infection in HIVinfected adults. AIDS 2001; 15: 2137-2147.

83 The Aurum Institute and London School of Hygiene \& Tropical Medicine. Media Brief: Thibela TB: effect of community-wide isoniazid preventive therapy on tuberculosis among gold miners in South Africa TB in South African gold mines. www.auruminstitute.org/documents/Thibela_TB_media_brief_20120308.pdf Date last accessed: March 2012. Date last updated: March 8, 2012.

84 Samandari T, Agizew TB, Nyirenda S, et al. 6-month versus 36-month isoniazid preventive treatment for tuberculosis in adults with HIV infection in Botswana; a randomised, doubleblind, placebo-controlled trial. Lancet 2011; 377: 1588-1598.

85 Lawn SD, Wood R. Short-course untargeted isoniazid preventive therapy in South Africa: time to rethink policy? Int J Tuberc Lung Dis 2012; 16: 995-996.

86 Golub JE, Chaisson RE, Martinson NA, et al. Additive effects of isoniazid preventive therapy and HAART. AIDS 2009; 23: 1446-1447.

87 Churchyard GJ, Fielding K, Charalambous S, et al. Efflcacy of secondary isoniazid preventive therapy among HIVinfected Southern Africans: time to change policy? AIDS 2003; 17: 2063-2070.

88 LoBue PA, Enarson DA, Thoen TC. Tuberculosis in humans and its epidemiology, diagnosis and treatment in the United States. Int J Tuberc Lung Dis 2010; 14: 1226-1232.

89 European Centre for Disease Prevention and Control/WHO Regional Office for Europe. Tuberculosis surveillance and monitoring in Europe 2012. Stockholm, European Centre for Disease Prevention and Control. 2012.

90 Moore-Gillon J, Davies PD, Ormerod LP. Rethinking TB screening: politics, practicalities and the press. Thorax 2010; 65: 663-665.

91 Thorpe LE, Laserson K, Cookson S, et al. Infectious tuberculosis among newly arrived refugees in the United States. N Engl J Med 2004; 350: 2105-2106.

92 Maloney SA, Fielding KL, Laserson KF, et al. Assessing the performance of overseas tuberculosis screening programs: a study among US-bound immigrants in Vietnam. Arch Intern Med 2006; 166: $234-240$.

93 Linas BP, Wong AY, Freedberg KA, et al. Priorities for screening and treatment of latent tuberculosis infection in the United States. Am J Respir Crit Care Med 2011; 184: 590-601.

94 Koster BFPJ, Meijer-Veldman W, van Loenhout-Rooyackers JH, et al. Role of the QuantiFERONß-TB Gold InTube assay in screening new immigrants for tuberculosis infection. Eur Respir J 2012; 40: 1443-1449.

95 National Institute for Health and Clinical Excellence (NICE). Tuberculosis: Clinical Diagnosis and Management of Tuberculosis, and Measures for its Prevention and Control. NiICE Clinical Guideline No 117. Manchester, NICE, 2006.

96 National Collaborating Centre for Chronic Conditions. Tuberculosis: Clinical diagnosis and management of tuberculosis, and measures for its prevention and control. London, United Kingdom, Royal College of Physicians, 2006.

97 Hardy AB, Varma R, Collyns T, et al. Cost-effectiveness of the NICE guidelines for screening for latent tuberculosis infection: the QuantiFERON-TB Gold IGRA alone is more cost-effective for immigrants from high burden countries. Thorax 2010; 65: 178-180.

98 Pareek M, Watson JP, Ormerod LP, et al. Screening of immigrants in the UK for imported latent tuberculosis: a multicentre cohort study and cost-effectiveness analysis. Lancet Infect Dis 2011; 11: 435-444.

99 Pareek M, Baussano I, Abubakar I, et al. Evaluation of immigrant tuberculosis screening in industrialized countries. Emerg Inf Dis 2012; 18: 1422-1429.

100 Cain KP, Garman KN, Laserson KF, et al. Moving towards tuberculosis elimination: implementation of statewide targeted tuberculin testing in Tennessee. Am J Respir Crit Care Med 2012; 186: 273-279.

101 Baker MA, Lin HH, Chang HY, et al. The risk of tuberculosis disease among persons with diabetes mellitus: a prospective cohort study. Clin Infect Dis 2012; 54: 818-825.

102 Corris V, Unwin N, Critchley J. Quantifying the association between tuberculosis and diabetes in the US: a casecontrol analysis. Chronic Illn 2012; 8: 121-134.

103 Young F, Wotton CJ, Critchley JA, et al. Increased risk of tuberculosis disease in people with diabetes mellitus: record-linkage study in a UK population. J Epidemiol Community Health 2012; 66: 519-523.

104 American Thoracic Society, Centers for Disease Control and Prevention, Infectious Diseases Society of America: controlling tuberculosis in the United States. Am J Respir Crit Care Med 2005; 172: 1169-1227. 
105 National Collaborating Centre for Chronic Conditions and the Centre for Clinical Practice at the National Institute for Health and Clinical Excellence. Tuberculosis (CG117). Clinical diagnosis and management of tuberculosis, and measures for its prevention and control. London, Royal College of Physicians, 2011.

106 Harries AD, Lin Y, Satyanarayana S, et al. The looming epidemic of diabetes-associated tuberculosis: learning lessons from HIV-associated tuberculosis. Int I Tuberc Lung Dis 2011; 15: 1436-1444.

107 Stevenson CR, Forouhi NG, Roglic G, et al. Diabetes and tuberculosis: the impact of the diabetes epidemic on tuberculosis incidence. BMC Public Health 2007; 7: 234.

108 Walker C, Unwin N. Estimates of the impact of diabetes on the incidence of pulmonary tuberculosis in different ethnic groups in England. Thorax 2010; 65: 578-581.

109 Bumbacea D, Arend SM, Eyuboglu F, et al. The risk of tuberculosis in transplant candidates and recipients: A TBNET consensus statement. Eur Respir J 2012; 40: 990-1013.

110 Wallis RS, Broder MS, Wong JY, et al. Granulomatous infections diseases associated with tumor necrosis factor antagonists. Clin Infect Dis 2004; 38: 1261-1265.

111 Carmona L, Gómez-Reino JJ, Rodríguez-Valverde V, et al. Effectiveness of recommendations to prevent reactivation of latent tuberculosis infection in patients treated with tumor necrosis factor antagonists. Arthritis Rheum 2005; 52: 1766-1772.

112 Ubel PA, Hirth RA, Chernew ME, et al. What is the price of life and why doesn't it increase at the rate of inflation? Arch Intern Med 2003; 163: 1637-1641.

113 Nienhaus A, Schablon A, Costa JT, et al. Systematic review of cost and cost-effectiveness of different TB-screening strategies. BMC Health Serv Res 2011; 11: 247.

114 Gourevitsch MN, Alcabes P, Wassermann WC, et al. Cost-effectiveness of directly observed chemoprophylaxis of tuberculosis among drug users at high risk for tuberculosis. Int J Tuberc Lung Dis 1998; 2; 531-540.

115 Becerra MC, Appleton SC, Franke MF, et al. Tuberculosis burden in households of patients with multidrug-resistant and extensively drug-resistant tuberculosis: a retrospective cohort study. Lancet 2011; 377: $147-152$.

116 Diel R, Rutz S, Castell S, et al. Tuberculosis: cost of illness in Germany. Eur Respir J 2012; 40: 143-151.

117 Floyd K, Hutubessy R, Kliiman K, et al. Cost and cost-effectiveness of multidrug-resistant tuberculosis treatment in Estonia and Russia. Eur Respir J 2012; 40: 133-142.

118 Van der Werf MJ, Sandgren A, Manissero D. Management of contacts of multidrug-resistant tuberculosis patients in the European Union and European Economic Area. Int J Tuberc Lung Dis 2012; 16: 426.

119 Seddon JA, Godfrey-Faussett P, Hesseling AC, et al. Management of children exposed to multidrug-resistant Mycobacterium tuberculosis. Lancet Infect Dis 2012; 12: 469-479.

120 van der Werf MJ, Langendam MW, Sandgren A, et al. Lack of evidence to support policy development for management of contacts of multidrug-resistant tuberculosis patients: two systematic reviews. Int J Tuberc Lung Dis 2012; 16: 288-296.

121 Management of persons exposed to multidrug-resistant tuberculosis. MMWR Recomm Rep 1992; 41: 61-71.

122 Department of Health, Republic of South Africa. Management of Drug-Resistant Tuberculosis: Policy Guidelines 2011. Pretoria: Ministry of Health, 2011.

123 European Centre for Disease Prevention and Control. Management of contacts of MDR TB and XDR TB patients. Stockholm, European Centre for Disease Prevention and Control, 2012.

124 Fraser A, Paul M, Attamna A, et al. Drugs for preventing tuberculosis in people at risk of multiple-drug-resistant pulmonary tuberculosis. Cochrane Database Syst Rev 2006; 2: CD005435.

125 Migliori GB, Zellweger JP, Abubakar I, et al. European Union Standards for Tuberculosis Care. Eur Respir J 2012; 39: 807-819.

126 Dharmadhikari AS, Mphahlele M, Stoltz A, et al. Surgical face masks worn by patients with multidrugresistant tuberculosis: impact on infectivity of air on a hospital ward. Am J Respir Crit Care Med 2012; 185: 1104-1149.

127 Stop TB. Partnership. The global plan to stop TB 2011-2015: transforming the fight towards elimination of tuberculosis. WHO/HTM/STB/2010.2. Geneva, World Health Organization, 2010.

128 Young DB, Gideon HP, Wilkinson RJ. Eliminating latent tuberculosis. Trends Microbiol 2009; 17: 183-188.

129 Sandgren A, Hollo V, Huitric E, et al. Epidemiology of tuberculosis in the EU/EEA in 2010: monitoring the progress towards tuberculosis elimination. Euro Surveill 2012; 17: pii, 20124.

130 Regional Committee for Europe. Roadmap for the Implementation of the Consolidated Action Plan to Prevent and Combat Multidrug- and Extensively Drug-Resistant Tuberculosis in the WHO European Region 2011-2015. EUR/ RC61/Inf.Doc./3. Copenhagen, World Health Organization Regional Office for Europe, 2011.

131 Health Protection Agency. Meeting of the HPA Board 30 September 2009. Tuberculosis Programme Update. www. hpa.org.uk/webc/HPAwebFile/HPAweb_C/1253205417019 Date last accessed: June 28, 2013. Date last updated: September 30, 2009.

132 Centers for Disease Control and Prevention. National TB program objectives and performance targets for 2015. www.cdc.gov/tb/programs/evaluation/indicators/default.htm Date last updated: September 1, 2012. Date last accessed June 28, 2013.

133 Kumar P. Journey of tuberculosis control movement in India: National tuberculosis programme to revised national tuberculosis control programme. Indian J Tuberc 2005; 52: 63-71.

134 Dye C. Practical preventive therapy for tuberculosis? N Engl J Med 2011; 365: 2230-2231.

135 MacIntyre CR, Plant AJ, Yung A, et al. Missed opportunities for the prevention of tuberculosis in Victoria, Australia. Int J Tuberc Lung Dis. 1997; 1: 135-141.

136 Ottenhoff THM, Ellner JJ, Kaufmann SHE. Ten challenges for TB biomarkers. Tuberculosis (Edinb) 2012; 92: Suppl. 1, S17-S20.

137 Sotgiu G, Centis R, D’Ambrosio L, et al. Efficacy, safety and tolerability of linezolid containing regimens in treating MDR-TB and XDR-TB: systematic review and meta-analysis. Eur Respir J 2012; 40: 1430-1442.

138 De Lorenzo S, Alffenaar JW, Sotgiu G, et al. Efficacy and safety of meropenem-clavunate added to linezolidcontaining regimens in the treatment of MDR-/XDR-TB. Eur Respir J 2013; 41: 1386-1392.

139 Alsaad N, van Altena R, Pranger AD, et al. Evaluation of co-trimoxazole in treatment of multidrug-resistant tuberculosis. Eur Respir J 2012 [In press DOI: 10.1183/09031936.00114812]. 
140 Zumla A, Blasi F, Raviglione MC. Rational use of anti-tuberculosis drugs in the EU: better patient care and less drug resistance. Eur Respir J 2012; 39: 802-804.

141 Raviglione MC, Lange C, Migliori GB. Preventing and managing antimicrobial resistance: imperative for chest physicians. Eur Respir J 2011; 37: 978-81.

142 European Centre for Disease Prevention and Control. Progressing Towards TB Elimination. A follow-up to the Framework Action Plan to Fight Tuberculosis in the European Union. Stockholm, European Centre for Disease Prevention and Control, 2010. 